\title{
DLC2 operates as a tumor suppressor gene in breast cancer via the RhoGTPase pathway
}

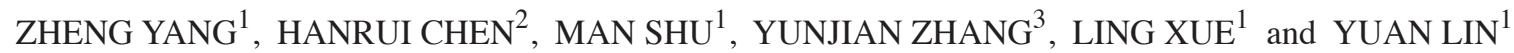 \\ ${ }^{1}$ Department of Pathology, The First Affiliated Hospital, Sun Yat-Sen University, Guangzhou, Guangdong 510080; \\ ${ }^{2}$ Department of Oncology, The First Affiliated Hospital, Guangzhou University of Chinese Medicine, \\ Guangzhou, Guangdong 510405; ${ }^{3}$ Department of Thyroid, Breast Surgery, The First Affiliated Hospital, \\ Sun Yat-Sen University, Guangzhou, Guangdong 510080, P.R. China
}

Received December 20, 2017; Accepted September 21, 2018

DOI: $10.3892 / 01.2018 .9874$

\begin{abstract}
Deleted in liver cancer 2 (DLC2) is a tumor suppressor, associated with various types of cancer. The aim of the present study was to analyze the expression of DLC2 in breast cancer, its clinical significance and its effect on breast cancer cell behavior. The expression of DLC2 was evaluated by immunohistochemistry in 131 cases of breast cancer. Associations among DLC2 expression and clinicopathological features were analyzed, and its effects on proliferation, motility, migration and invasion in DLC2-knockdown breast cancer cell lines were observed. The results indicated that DLC2 was expressed in $42.75 \%$ of breast cancer cases (56/131) and in $79.39 \%$ of adjacent normal tissues (104/131). Lower expression of DLC2 in breast cancer was associated with tumor differentiation $(\mathrm{P}<0.001)$, lymph node metastasis $(\mathrm{P}<0.001)$ and poor prognosis $(\mathrm{P}<0.001)$. The silencing of the DLC2 gene in human breast cancer cell indicated an increased number of cells entering $\mathrm{S}$ phase, and increased abilities of clone formation, cell migration and invasion. Downregulated expression of DLC2 was associated with activated Ras homolog family member A and decreased Rac family small GTPase 1, cell division cycle 42 and Rho-associated protein kinase- 2 expression levels, indicating that DLC2 may serve a regulatory function in breast cancer cell proliferation and invasion via the RhoGTPase pathway. The results of the present study suggested that DLC2
\end{abstract}

Correspondence to: Dr Yuan Lin or Professor Ling Xue, Department of Pathology, The First Affiliated Hospital, Sun Yat-Sen University, 2 Zhongshang Road, Guangzhou, Guangdong 510080, P.R. China

E-mail: liny36@mail.sysu.edu.cn

E-mail: xuel@mail.sysu.edu.cn

Abbreviations: DLC2, deleted in liver cancer 2; TMA, tissue microarray; FBS, fetal bovine serum; DMEM, Dulbecco's modified Eagle's medium; Rock, Rho-associated protein kinase; OS, overall survival

Key words: deleted in liver cancer 2, breast cancer, tumor suppressor, proliferation, anti-Ras homolog family member A serves as a suppressor gene in the development of breast cancer and may be a prognostic marker for patients with breast cancer.

\section{Introduction}

The increase in early diagnosis rate, in addition with emerging treatments and targeting medicine, have decreased the mortality rate of patients with breast cancer in a number of developed countries $(1,2)$. However, the incidence of breast cancer is increasing in the majority of countries, including China (3). The primary causes of mortality in breast cancer are recurrence and metastasis (4), whereas angiogenesis serves an important function in the process of breast cancer disease occurrence, recurrence and metastasis $(5,6)$.

Deleted in liver cancer 2 (DLC2), also named StAR-related lipid transfer domain 13, was successfully cloned by Ching et al in 2003 (7). As an adhesion molecule, DLC2 lies on the adhesion plaque of the cell membrane and is involved in cell adhesion (7), mitosis (8) and sensory nerve conduct (9) under physiological conditions. DLC2 is poorly expressed in a variety of tumors, including liver cancer, lung cancer, colon cancer and rectal cancer (10), and is considered to be a potential tumor suppressor gene $(7,10)$. In a previous study, a DLC2-knockout mouse model was established and it was indicated that DLC2-knockout mice exhibited more marked angiogenesis ability compared with that of wild-type mice (11). In addition, it was indicated that DLC2 was downregulated in $\sim 50 \%$ of cases of breast cancer at the mRNA level (data not shown). This suggests that, as an adhesion-associated molecule, DLC2 may be involved in tumor formation and metastasis in breast cancer.

In the present study, the expression of DLC2 in breast cancer was investigated, and its association among clinicopathological parameters and the survival of patients was examined. The effect of DLC2 on proliferation, migration and invasion of breast cancer cells was additionally observed, in an attempt to determine the function of DLC2 in the carcinogenesis and development of breast cancer.

\section{Materials and methods}

Patients and tissue specimens. A total of 131 paraffin-embedded surgical specimens of breast cancer were collected at The First 
Affiliated Hospital of Sun Yat-sen University (Guangzhou, China) between January 2007 and December 2007 for immunohistochemical assay. All specimens were from primary tumors. All patients were female and the median age of the patients was 43 years (range, 33-79 years). The ethics committee at The First Affiliated Hospital of Sun Yat-sen University (Guangzhou, China) approved the present study. The clinicopathological data are presented in Table I. All patients were diagnosed with primary breast cancer, and none of the patients received any form of medical treatment prior to surgery. All patients provided written informed consent prior to participation in the present study.

Construction of tissue microarrays (TMAs). A tissue array device (Unitma Quick-Ray; Utigma Co., Ltd., Seoul, South Korea) was used to construct the TMAs. For each case, two 1-mm-diameter cylinders selected from two different areas were included in the TMAs. Finally, 4 TMA blocks were constructed, each containing 120 cylinders. Consecutive $4-\mu \mathrm{m}$-thick sections were cut using a microtome and air-dried overnight at room temperature.

Immunohistochemistry. The tissue samples were fixed win $10 \%$ phosphate-buffered formalin for $6-48 \mathrm{~h}$ at room temperature. Sections (4- $\mu \mathrm{m}$-thick) were cut from the TMA blocks. All sections were deparaffinized with xylene and rehydrated by a graded ethanol series to distilled water. The sections were then heated for antigenic retrieval in sodium citrate buffer ( $\mathrm{pH}$ 6.0; WeijiangGene Chem Co., Ltd., Guangzhou, China) for $3 \mathrm{~min}$ at $100^{\circ} \mathrm{C}$. Endogenous peroxidase was subsequently blocked with $0.3 \% \mathrm{H}_{2} \mathrm{O}_{2}$ for $15 \mathrm{~min}$ at room temperature and incubated with 5\% normal goat serum (Shanghai GeneChem Co., Ltd., Shanghai, China) in PBS for 30 min at room temperature. The slides were incubated with rabbit monoclonal anti-DLC2 antibody (catalog no. ab126489; 1:150; Abcam, Cambridge, MA, USA) overnight at $4^{\circ} \mathrm{C}$. Following three washes with PBS, the slides were incubated with anti-rabbit secondary antibody (catalog no. 3900s; 1:200; Cell Signaling Technology Inc., Danvers, MA, USA) for $1 \mathrm{~h}$ at room temperature. Subsequently, the Non-Biotin HRP Detection system (Shanghai GeneChem Co., Ltd., Shanghai, China) was used, according to the manufacturer's protocol. Diaminobenzidine (WeijiangGene Chem Co., Ltd.) was used for color reaction, and the antibody was replaced by normal goat serum for negative controls. Slides were reviewed and scored using a light microscope (magnification, $\mathrm{x} 400$ ) by two independent pathologists who were blinded to the data and affiliated to the Department of Pathology, The First Affiliated Hospital, Sun Yat-Sen University (Guangzhou, China). Scores given by the two pathologists were averaged for further comparative evaluation of DLC2 expression. The slides were counterstained with haematoxylin at room temperature for $1 \mathrm{~min}$. Immunoreactivity was scored according to staining intensity of the product as follows: 0 , no staining; 1 , weak staining (light yellow); 2, moderate staining (yellow brown) and 3 , strong staining (brown). The percentage of stained cells was classified as follows: 0 , no staining; $1,1-25 \%$ staining; $2,26-75 \%$ staining and $3,>75 \%$ staining. Final scores $>4$ were defined as positive and scores $\leq 4$ as negative (12).
Cell culture. The human breast cancer cell lines MDA-MB-231 and MDA-MB-468 were obtained from the American Type Culture Collection (Manassas, VA, USA). MDA-MB-231 cells were cultured in RPMI-1640 medium supplemented with $10 \%$ fetal bovine serum (FBS; Gibco; Thermo Fisher Scientific, Inc., Waltham, MA, USA) and MDA-MB-468 cells were cultured in Dulbecco's modified Eagle's medium (DMEM) supplemented with $10 \%$ FBS. All cells were cultured at $37^{\circ} \mathrm{C}$ with $5 \% \mathrm{CO}_{2}$ for $24 \mathrm{~h}$. All cells were used within 2 months following receipt or resuscitation of frozen aliquots.

Cell transfection with vectors. Cells were incubated in a humid incubator at $37^{\circ} \mathrm{C}$ with $5 \% \mathrm{CO} 2$ for $24 \mathrm{~h}$, Stable DLC2-knockdown MDA-MB-468 and MDA-MB-231 cell lines were constructed. A total of $5 \mu \mathrm{g} / \mathrm{ml} \mathrm{GV} 115$ plasmid (catalog no. pGCSIL-004; Shanghai GeneChem Co., Ltd.) was used. Transfection into cells at 30-50\% confluency was performed with Lipofectamine 2000 (Invitrogen; Thermo Fisher Scientific), according to the manufacturer's protocol. Stable short hairpin (sh)DLC2 or sh green fluorescent protein (GFP) cells were generated by infection with shRNA lenti viruses against DLC2 or GFP (Shanghai GeneChem Co., Ltd.), followed by puromycin $(2.5 \mu \mathrm{g} / \mathrm{ml})$ selection. The expression level of DLC2 in stable shDLC2 or shGFP breast cancer cells was verified by western blot analysis following $48 \mathrm{~h}$.

Cell cycle analysis. Cell cycle content was determined by flow cytometry. Both cell lines were collected at $10^{4}$ cells/well and fixed in $70 \%$ cold ethanol for $24 \mathrm{~h}$ at room temperature. Fixed cells were subsequently washed three times with PBS followed by staining with $50 \mu \mathrm{g} / \mathrm{ml}$ propidium iodide (catalog no. P1304MP; Thermo Fisher Scientific, Inc.) for $30 \mathrm{~min}$ protected from light at room temperature. Stained cells were analyzed with an Accuri C6 Plus flow cytometer (version 3.0; BD Biosciences, Franklin Lakes, NJ, USA) and data were obtained based on the analysis of 12,000 cells.

Plate clone formation assay. In total, 200 cells/well of each cell line were seeded in 6-well plates (Corning Incorporated, Corning, NY, USA). Cells were cultured in DMEM supplemented with $10 \%$ FBS for 14 days at $37^{\circ} \mathrm{C}$ PBS was flushed twice over $4 \%$ paraformaldehyde-fixed cells after $15 \mathrm{~min}$ at room temperature, followed by $30 \mathrm{~min}$ of $4 \%$ Giemsa staining at room temperature. The number of clones formed was determined using a light microscope (magnification, 400x) and ten fields of view were randomly selected. ImageJ software (v1.8.0; National Institutes of Health, Bethesda, MD, USA) was used for quantification.

Soft agar assay. Both cell lines (5,000/well) were suspended in medium containing $15 \%$ FBS and $0.5 \%$ agarose and seeded on a layer of solidified agarose in each $60 \times 15 \mathrm{~mm}$ cell-culture dish (Corning Incorporated). Following 28 days, colony-forming units with cell numbers $\geq 15$ were counted and colony-forming efficiency was calculated as the number of colonies/the number of seeded cells. To view ten randomly selected fields of view a light microscope (magnification, $\mathrm{x} 400$ ) was used. ImageJ software (v1.8.0, National Institutes of Health) was used to analyze data. 
Table I. Association between DLC2 and clinicopathological parameters.

\begin{tabular}{|c|c|c|c|c|}
\hline \multirow[b]{2}{*}{ Clinicopathological parameter } & \multirow[b]{2}{*}{ No. of patients (\%) } & \multicolumn{3}{|c|}{ DLC2, no. of patients } \\
\hline & & - & + & P-value \\
\hline Median age, years & & & & 0.762 \\
\hline$\leq 43$ & $78(59.5)$ & 43 & 44 & \\
\hline$>43$ & $53(40.5)$ & & & \\
\hline Tumor size, cm & & & & 0.417 \\
\hline $\mathrm{T} 1(\leq 2)$ & $66(50.4)$ & 33 & 33 & \\
\hline $\mathrm{T} 2(2-5)$ & $42(32.1)$ & 27 & 15 & \\
\hline $\mathrm{T} 3(>5)$ & $23(17.5)$ & 15 & 8 & \\
\hline Lymphatic metastasis & & & & $<0.001^{\mathrm{a}}$ \\
\hline N0 & $82(62.6)$ & 30 & 52 & \\
\hline N1-N3 & $49(37.4)$ & 45 & 4 & \\
\hline Tumor differentiation ${ }^{\mathrm{b}}$ & & & & $<0.001^{\mathrm{a}}$ \\
\hline I & $32(24.4)$ & 6 & 26 & \\
\hline II & $34(26.0)$ & 14 & 20 & \\
\hline III & $65(49.6)$ & 55 & 10 & \\
\hline Ki67 & & & & 0.085 \\
\hline$<14$ & $52(39.7)$ & 25 & 27 & \\
\hline$\geq 14$ & $79(60.3)$ & 50 & 29 & \\
\hline ER & & & & $<0.001^{\mathrm{a}}$ \\
\hline$+(\geq 1)$ & $35(26.7)$ & 9 & 30 & \\
\hline$-(<1)$ & $96(73.3)$ & 66 & 26 & \\
\hline PR & & & & 0.622 \\
\hline$+(\geq 1)$ & $110(84.0)$ & 64 & 46 & \\
\hline$-(<1)$ & $21(16.0)$ & 11 & 10 & \\
\hline Her $2 /$ CerbB $^{c}$ & & & & 0.411 \\
\hline,-+ & $91(69.5)$ & 49 & 42 & \\
\hline++ & $14(10.7)$ & 10 & 4 & \\
\hline+++ & $26(19.8)$ & 16 & 10 & \\
\hline Histological type & & & & 0.546 \\
\hline Invasive ductal carcinoma & $113(86.3)$ & 62 & 51 & \\
\hline Intraductal carcinoma & $9(6.9)$ & 4 & 5 & \\
\hline Invasive lobular carcinoma & $2(1.5)$ & 2 & 0 & \\
\hline Special carcinoma & $7(5.3)$ & 7 & 0 & \\
\hline
\end{tabular}

${ }^{\mathrm{a}} \mathrm{P}<0.05 .{ }^{\mathrm{b}}$ According to the World Health Organization classification of tumors of the breast (13). ${ }^{\mathrm{c}}$ Values of CerbB2 were measured according to previously discussed guidelines (32). DLC2, deleted in liver cancer 2; ER, estrogen receptor; PR, progesterone receptor; Her2, human epidermal growth factor receptor 2.

Wound healing migration assay. Cells were seeded on 24-well plates and cultured to $100 \%$ confluence. The confluent monolayer was wounded with a yellow pipette tip and was allowed to migrate for $12 \mathrm{~h}$ for MDA-MB-468 cells and $20 \mathrm{~h}$ for MDA-MB-231 cells. Images were captured at 0 and $12 \mathrm{~h}$ in the same position for MDA-MB-468 cells, and at 0 and $20 \mathrm{~h}$ in the same position for MDA-MB-231 cells. Migration distances were measured, and speed was determined in $\mu \mathrm{m} / \mathrm{h}$ using Image J software (v1.8.0, National Institutes of Health).

Invasion assay. Matrigel (BD Biosciences) was dissolved at $4^{\circ} \mathrm{C}$ overnight. Cells were starved in serum-free medium
(Gibco; Thermo Fisher Scientific, Inc.) $24 \mathrm{~h}$ prior to assay. Cells were harvested, centrifuged at $252 \mathrm{x}$ g for $5 \mathrm{~min}$ at $4^{\circ} \mathrm{C}$ and resuspended three times with culture medium containing $1 \%$ FBS at room temperature, and subsequently brought to a concentration of $10^{6}$ cells $/ \mathrm{ml}$. Following rehydration, $250 \mu \mathrm{l}$ medium was removed from Transwell inserts and $250 \mu \mathrm{l}$ cell suspensions were added. Inserts were placed in a 24-well plate, and $500 \mu \mathrm{l}$ complete RPMI 1640 medium (Gibco; Thermo Fisher Scientic, Inc.), with 15\% FBS, containing $5 \mu \mathrm{g} / \mathrm{ml}$ fibronectin (R\&D Systems, Inc., Minneapolis, MN, USA), as an adhesive substrate was added to the lower wells. Complete RPMI 1640 medium $(500 \mu 1)$ with $10 \%$ serum 
was added to the upper wells. Plates were incubated for $24 \mathrm{~h}$ at $37^{\circ} \mathrm{C}$ in a $5 \% \mathrm{CO}_{2}$ incubator. The Transwells (Corning Incorporated) were removed from the 24-well plates. Cells were stained with crystal violet $(0.1 \%)$ for $30 \mathrm{~min}$ at room temperature and washed twice in PBS. Non-invasive cells were scraped off on the top of the Transwell with a cotton swab and the number of invading cells was counted under a light microscope (magnification, x400) in ten randomly selected fields of view.

Western blot analysis. Protein from both cell lines was dissolved in SDS lysis buffer (Shanghai GeneChem Co., Ltd.), and protein concentration was determined using a bicinchoninic acid protein assay kit (Shennengbocai Inc., Shanghai, China), according to the manufacturer's protocols. A total of $20 \mu \mathrm{g}$ of each protein sample was separated by SDS-PAGE ( $8 \%$ gel) and transferred onto polyvinylidene difluoride membranes overnight at $30 \mathrm{~V}$. The membranes were subsequently blocked with 5\% non-fat dry milk in PBS containing $0.1 \%$ Tween-20 for $1 \mathrm{~h}$ at room temperature and incubated with the following primary antibodies: Anti-DLC2 (ab126489; dilution, 1:2,000; Abcam), anti-Ras homolog family member A (RhoA; catalog no. ab54835; dilution, 1:2,000; Abcam), anti-Rac family small GTPase 1 (Rac-1; catalog no. ab33186; dilution, 1:2,000; Abcam), anti-cell division cycle 42 (Cdc42; catalog no. ab64533; dilution, 1:4,000; Abcam), anti-Rho-associated protein kinase (Rock)-1 (catalog no. ab45171; dilution, 1:8,000; Abcam), anti-Rock-2 (catalog no. MABN1037; dilution, 1:8,000; EMD Millipore, Billerica, MA, USA), anti-GAPDH (catalog no. 51332; dilution, 1:10,000; Cell Signaling Technology Inc.) overnight at $4^{\circ} \mathrm{C}$. Following incubation with the primary antibody, the membranes were washed three times with Tris-buffered saline containing Tween-20 and incubated with anti-rabbit secondary antibody (catalog no. 5127; dilution, 1:10,000; Cell Signaling Technology, Inc.) for $2 \mathrm{~h}$ at room temperature. The signal was detected by electrochemiluminescence (EMD Millipore). Images were captured on X-ray film and protein expression was quantified using ImageJ software (v1.8.0, National Institutes of Health).

Statistical analysis. All data are presented as the mean \pm standard deviation from three independent experiments. P-values were calculated by an unpaired Student's t-test or $\chi^{2}$ test using SPSS for Windows software (version 16.0; SPSS, Inc., Chicago, IL, USA). The Kaplan-Meier method was used to analyze disease-free survival time of patients and comparisons were analyzed by log-rank test. $\mathrm{P}<0.05$ was considered to indicate a statistically significant difference.

\section{Results}

DLC2 is downregulated in breast cancer and is associated with tumor differentiation and lymph node metastasis. The expression of DLC2 protein was detected by immunohistochemistry in TMAs containing 131 cases of breast cancer tissue and adjacent normal tissue. The clinicopathological data were collected and analyzed. The results indicated that the DLC2 expression level in breast cancer tissue was lower compared with the adjacent normal tissue $(\mathrm{P}<0.05$;
Table I; Fig. 1). The association between DLC2 expression and clinicopathogical parameters was analyzed and it was identified that lower expression of DLC2 in breast cancer tissue was significantly associated with tumor differentiation $(\mathrm{P}<0.001)$ and lymph node metastasis $(\mathrm{P}<0.001$; Table $\mathrm{I})$. The positive rate of DLC2 expression was $81.34 \%$ (26/32) in well differentiated grade I (13) breast cancer and was markedly higher $(\mathrm{P}>0.05)$ compared with poorly differentiated grade III breast cancer at $15.38 \%$ (10/65) (Table I). Cancer grade was determined using a cancer grading system as previously described (13). The positive rate of DLC2 expression in breast cancer with lymphatic metastasis was $8.26 \%$ (4/49) compared with $63.41 \%$ (52/82) in breast cancer without lymphatic metastasis (Table I). The results also indicated that the DLC2 expression was associated with the expression of estrogen receptor $(\mathrm{P}<0.001)$, which is important for the prognosis and treatment of breast cancer. However, there was no statistical significant difference between the expression of DLC2 and other clinical features, including age, histological type, tumor size and the expression of progesterone receptor, Ki-67 and CerbB2 (Table I).

Expression of DLC2 in breast cancer is associated with patient overall survival $(O S)$ rate. Following a median follow-up of 85 months (range, 22-136 months), 61 (53.4\%) patients experienced disease recurrence (Fig. 1E). Low expression of DLC2 was significantly associated with a poorer OS rate $(\mathrm{P}<0.001)$. Patients with negative DLC2 expression had a 10-year OS rate of $34.7 \%$ [95\% confidence interval (CI), 17.2-51.0], whereas those with positive DLC2 expression had a 10-year OS rate of 78.6\% (95\% CI, 62.65-93.9; $\mathrm{P}<0.001$; Fig. 1E). These results indicated that DLC2 expression may be an independent prognostic marker for OS rate in patients with breast cancer.

Knockdown of DLC2 promotes proliferation, migration and invasion in breast cancer cells. The aforementioned results of DLC2 expression in breast cancer tissue strongly indicate that DLC2 may serve as a tumor suppressor in the development of breast cancer. To analyze the function of DLC2 in breast cancer cells, DLC2-silencing (shDLC2) and control (shGFP) stable cell lines in MDA-MB-468 and MDA-MB-231 were generated. Flow cytometry was performed to analyze the cell cycle status of shDLC2 and shGFP stable cell lines. Results indicated that the number of cells entering $S$ phase significantly increased for the shDLC2 group compared with the shGFP group, when DLC2 was knocked down in MDA-MB-468 and MDA-MB-231 cells ( $\mathrm{P}<0.05$; Fig. 2A). In MDA-MB-468, cell plate cloning experiments indicated that the shDLC2 stable cell line exhibited significantly increased cell clone formation ability compared with the shGFP cell line ( $\mathrm{P}<0.05$; Fig. $2 \mathrm{~B})$. In addition, soft agar cloning experiments indicated that the colony forming efficiency was $1.98 \%$ in shDLC2 stable cell line, compared with $0.66 \%$ in the shGFP group $(\mathrm{P}<0.05$; Fig. 2C). Similar results were observed in MDA-MB-231 cells (Fig. 2B and C).

In wound healing migration assay, silencing of DLC2 in MDA-MB-468 and MDA-MB-231 cells significantly promoted cell invasion at 12 and $20 \mathrm{~h}$, respectively, following scratching ( $\mathrm{P}<0.05$; Fig. $3 \mathrm{~A}$ and $\mathrm{B})$. In addition, the Transwell invasion assay indicated that downregulation of DLC2 in 
A

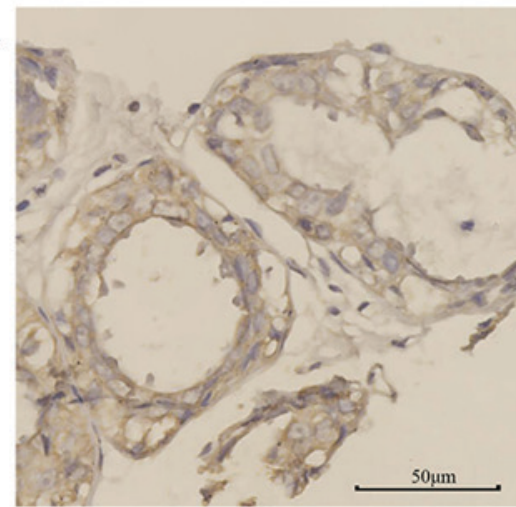

$\mathrm{C}$

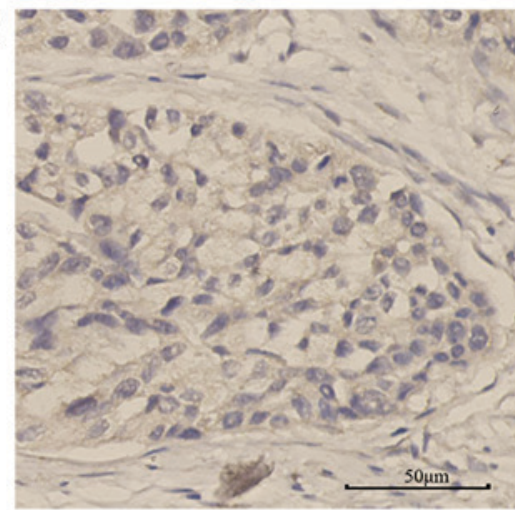

$\mathrm{B}$

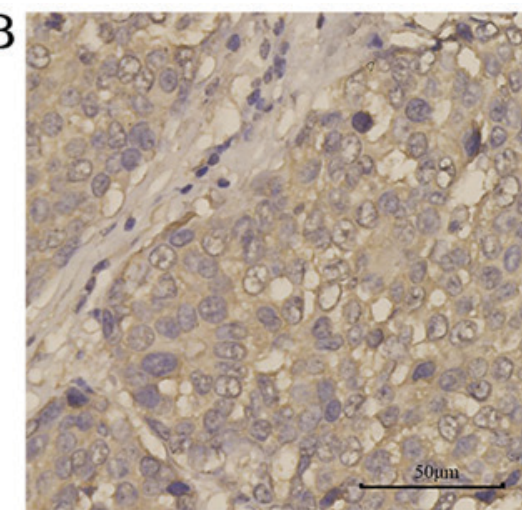

$\mathrm{D}$

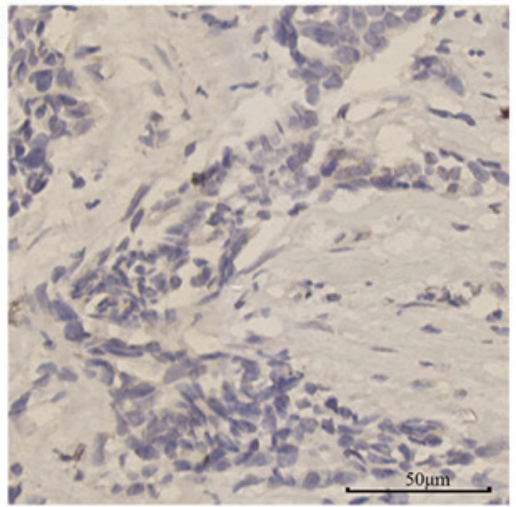

$\mathrm{E}$

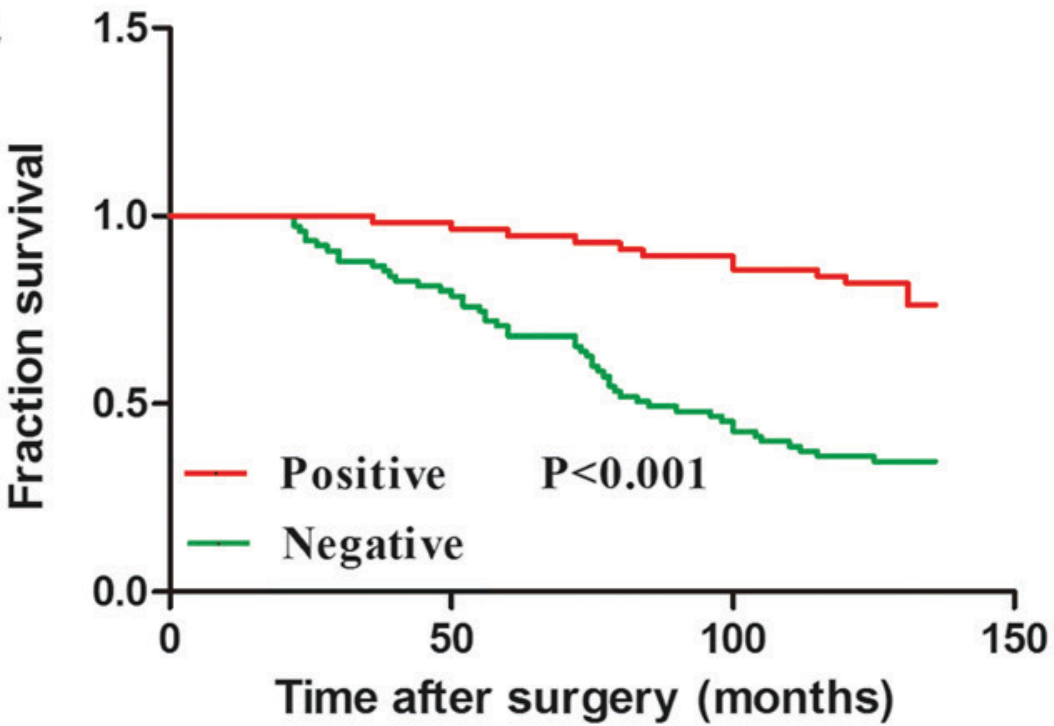

Figure 1. Expression pattern of DLC2 in breast cancer and respective survival analysis. Scale bar $=50 \mu \mathrm{m}$. (A) Staining of DLC2 in normal breast tissues. (B) High expression of DLC2 in breast cancer tissue. (C) Low expression of DLC2 in breast cancer tissue. (D) Low expression of DLC2 in breast cancer tissue with lymphatic metastasis. (E) Kaplan-Meier recurrence-free survival curve, according to DLC2 expression. Scale bar=50 $\mu \mathrm{m}$. DLC2, deleted in liver cancer 2 .

breast cancer cells significantly increased migration, particularly in MDA-MB-468 cells $(\mathrm{P}<0.05$; Fig. 3C).

DLC2 may regulate breast cancer cell proliferation and invasion via the RhoGTPase pathway. As DLC2 is a RhoGTPase activation protein-containing molecule, the present study investigated whether the observed results in DLC2-knockdown breast cancer cell lines were dependent on the RhoGTPase pathway. Western blot analysis indicated that the expression of RhoA was enhanced in the breast cancer cell lines MDA-MB-468 and MDA-MB-231, following knockdown of DLC2 (Fig. 4A). In addition, Rac-1, Cdc42, and Rock-2 were downregulated (Fig. 4A). However, the expression of Rock-1 exhibited no obvious change (Fig. 4A). Densitometry analysis indicated that DLC2 may regulate these cellular processes through the RhoGTPase pathway, including regulation of Rac-1, Cdc42, and Rock-2 (Fig. 4B and C).

\section{Discussion}

DLC2 has been identified as a tumor suppressor gene and is significantly downregulated in a wide range of human tumors $(7,10)$, including liver cancer, lung cancer, colon cancer and rectal cancer (8). In breast cancer, it has been reported 
A

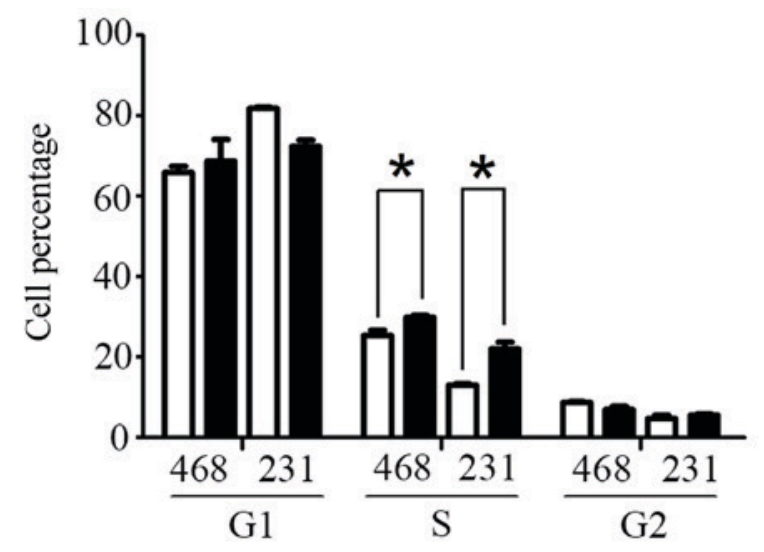

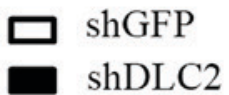

shDLC2
B

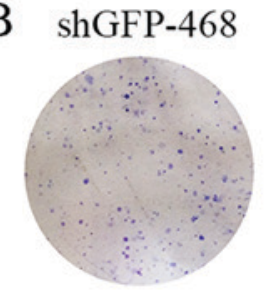

shGFP-231

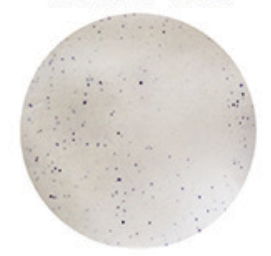

C $\quad$ shGFP-468

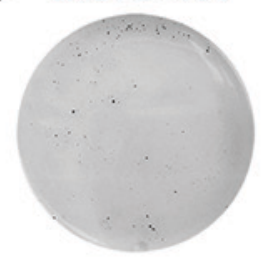

shGFP-231

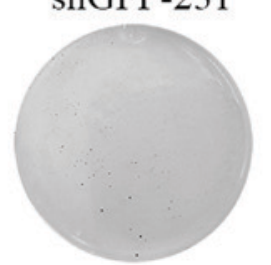

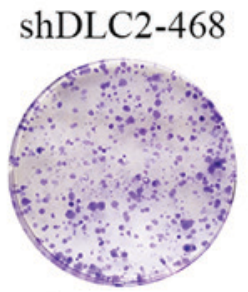

shDLC2-231

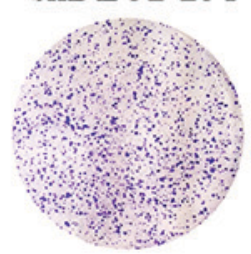

$\operatorname{shDLC2-468}$

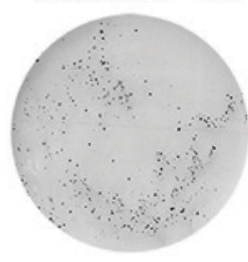

$\operatorname{shDLC2-231~}$

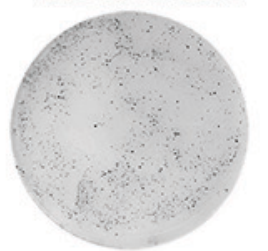

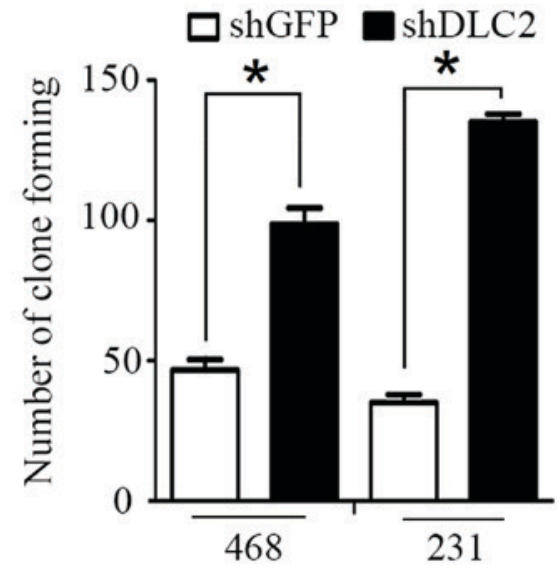

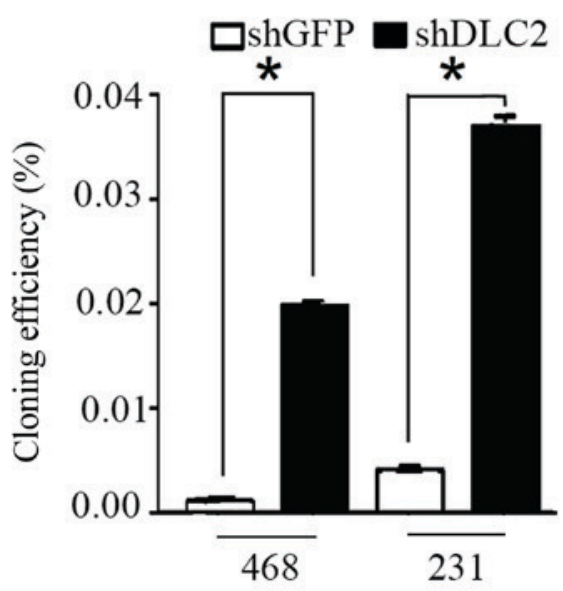

Figure 2. Influence of DLC2 expression in breast cancer on the biological behavior of cells. (A) Knockdown of DLC2 increased the number of cells to enter $\mathrm{S}$ phase. (B) Knockdown of DLC2 enhanced cancer cell clone formation ability and (C) soft agar cloning formation ability. * $\mathrm{P}<0.05$. DLC2, deleted in liver cancer 2; sh, short hairpin; GFP, green fluorescent protein.

that significant DLC2 downregulation was exhibited in $73 \%$ of tumors by reverse transcription-quantitative polymerase chain reaction (10). In the present study, DLC2 expression was detected in 131 cases of breast cancer by immunohistochemistry. The present study, to the best of our knowledge, has used the largest sample to investigate the protein expression of DLC2 in breast cancer. The results suggest that downregulation of DLC2 is associated with tumor differentiation, and is overall in agreement with previous reports $(10,14)$.
In addition, the results of the present study indicate that the downregulation of DLC2 is significantly associated with poor prognosis in patients with breast cancer. This indicates DLC2 may be a prognostic marker for patients with breast cancer. In addition, the results of the present study indicated that lower expression of DLC2 in breast cancer may promote proliferation and viability. Similar observations were reported previously in various tumor types. El-Sitt et al (15) reported that upregulation of DLC2 expression resulted in a limited 

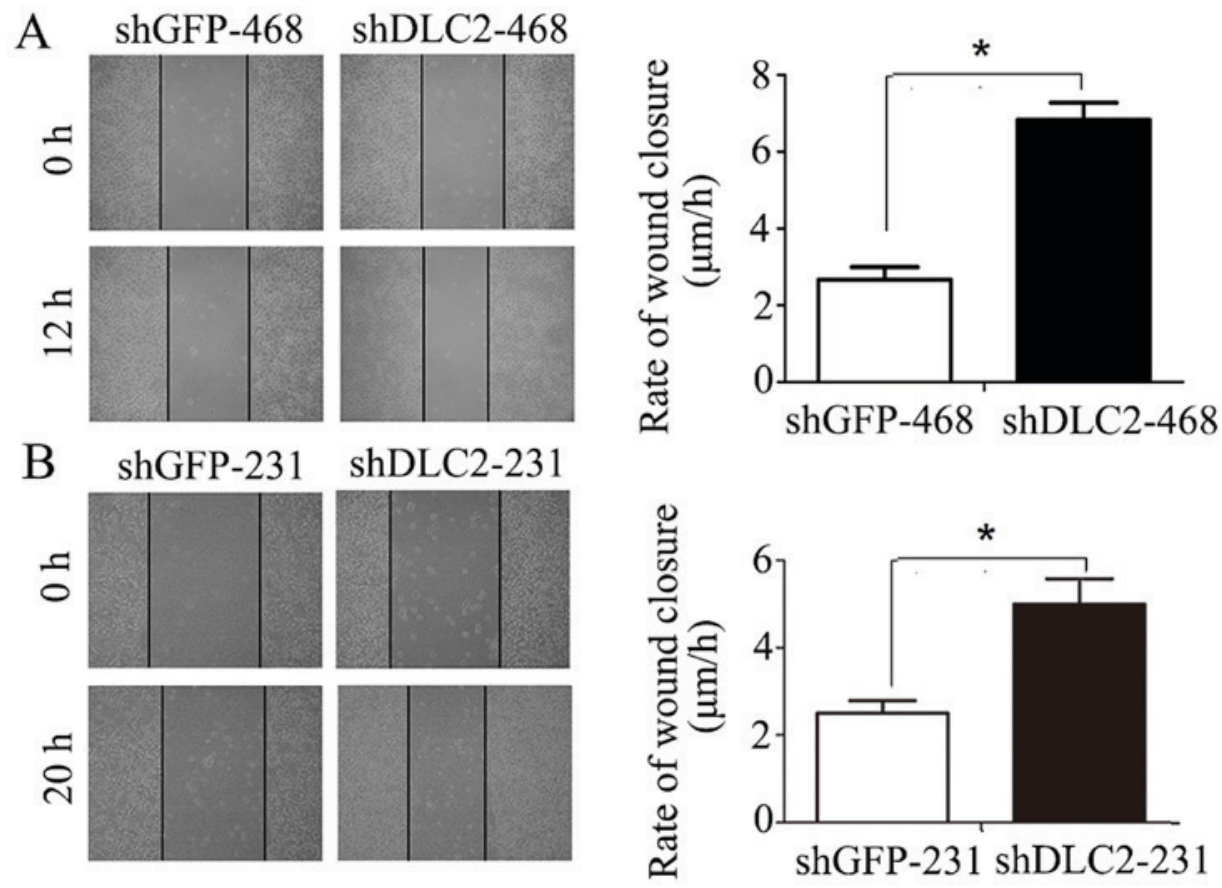

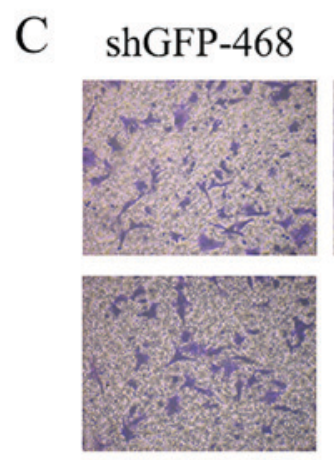

shGFP-231

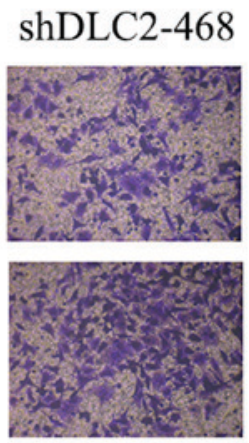

shDLC2-231

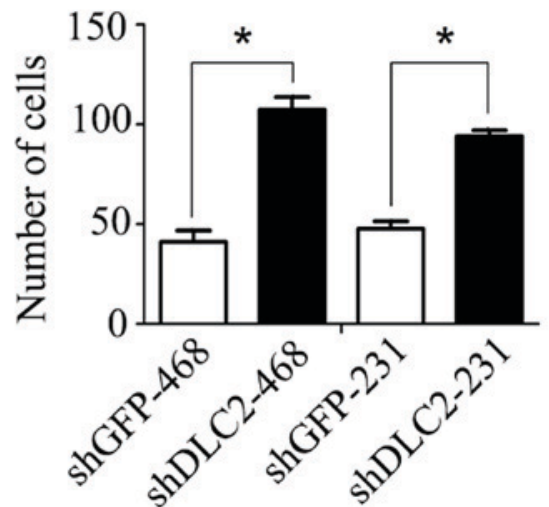

Figure 3. Influence of DLC2 expression in breast cancer on the biological behavior of cells. Knockdown of DLC2 in (A) MDA-MB-468 and (B) MDA-MB-231 enhanced cancer cell invasion. (C) Downregulation of DLC2 in breast cancer cells enhanced migration, particularly in MDA-MB-468 cells. * $<<0.05$. DLC2, deleted in liver cancer 2; sh, short hairpin; GFP, green fluorescent protein.

number of astrocytoma cells in $\mathrm{S}$ and $\mathrm{G}_{2}$ phase. The studies by Leung et al $(16,17)$ reported that silencing of DLC2 in liver cancer cells led to an increased proliferation rate. In addition, studies have demonstrated that DLC1 and DLC3, DLC family members together with DLC2, are also involved in the regulation of proliferation (15,18-22). These results indicate that DLC2 serves as a tumor suppressor in tumorigenesis.

Furthermore, the results of the present study indicated that DLC2 affected tumorigenesis, and influenced metastasis in breast cancer. It was indicated that decreased expression of DLC2 was associated with breast cancer metastasis. However, the results of the present study, in addition to those of previous studies $(14,23)$, indicated that downregulation of DLC2 increased the migratory and invasive ability of breast cancer cells. We hypothesized that this was associated with DLC2 function in focal adhesion, which is required for cell movement, and that the deletion of DLC2 may cause this change in cell movement. This may suggest that DLC2 participates in tumor metastasis. In our previous study, it was identified that DLC2 was involved in angiogenesis (11). Angiogenesis is important for tumor metastasis (6), therefore providing additional evidence that DLC2 may be involved in tumor angiogenesis and regulate tumor metastasis in breast cancer. DLC1, a DCL family member, was reported to regulate tumor angiogenesis in prostate cancer, through the hypoxia-inducible factor 1-vascular endothelial growth factor pathway (24). However, the underlying molecular mechanism of DLC2 remains unknown.

DLC2 belongs to the RhoGTPase activation protein family, and the members of this family primarily regulate RhoGTPase, including RhoA, Rac1, Cdc42 and Rock expression (25). In our previous research, it was indicated that DLC2 regulated angiogenesis depending on RhoA (9). Alternative studies have also demonstrated that DLC2 serves an important function through the RhoA pathway. It has been reported that, during pancreatic development, Rho signaling was restricted by DLC2 (26). In liver cancer, DLC2 has been reported to suppress tumor cell proliferation and colony formation in a RhoGAP-dependent manner (17). In astrocytoma, the regulation of RhoA in focal adhesion was essential 
A
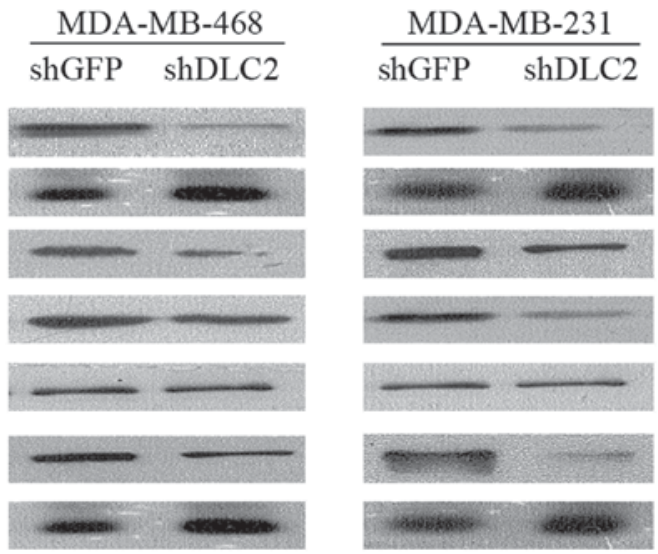

DLC2

RhoA

Rac-1

$\mathrm{Cdc} 42$

Rock-1

Rock-2

GAPDH

B
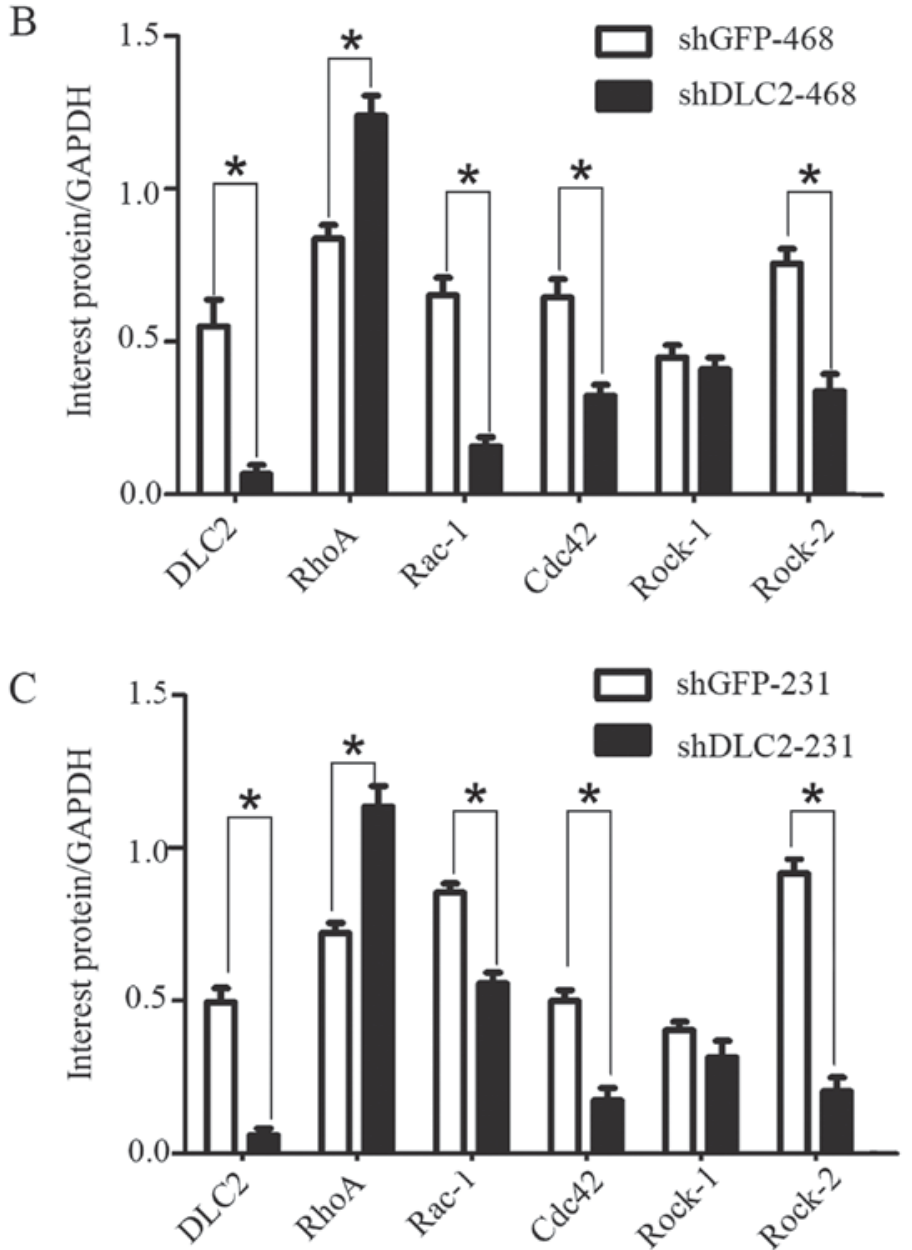

Figure 4. Silencing of DLC2 in breast cancer cells promotes expression of RhoA and inhibits expression of Rac-1, Cdc42 and Rock-2. (A) Expression level of DLC2 protein and quantitative analysis in (B) MDA-MB-468 and (C) MDA-MB-231 cells. "P<0.05. DLC2, deleted in liver cancer 2; sh, short hairpin; GFP, green fluorescent protein; Rock, Rho-associated protein kinase; RhoA, Ras homolog family member A; Rac-1, Rac family small GTPase 1; Cdc42, cell division cycle 42.

in cell motility (27). In addition, in breast cancer, it was indicated that miR-125b affected the metastatic activities of cells. The regulation of $\alpha$-smooth muscle actin by miR-125b has been reported to be dependent on the DLC2-RhoA-Rock signaling pathway (28). In addition, Kaplan-Meier survival analysis performed in a previous study that the mRNA expression of DLC2 and its competing endogenous (ce)RNAs, including cadherin-5 (CDH5), homeobox D1 (HOXD1) and homeoboxD10 (HOXD10), is associated with survival rate of patients with breast cancer. DLC2 3' untranslated region (UTR) suppressed metastasis via inhibiting epithelial-mesenchymal transition (EMT) in breast cancer (29). It was also indicated that the $\mathrm{C}-\mathrm{C}$ motif chemokine receptor 2 (CCR2) 3' UTR inhibited cell metastasis, by repressing EMT. The CCR2 3' UTR has also been reported to serve as a metastatic suppressor by acting as a ceRNA for DLC2 and therefore, inhibiting the RhoA-Rock-1-MLC-filamentous actin pathway in breast cancer cells (30). In addition, the DLC2 3' UTR 
has been demonstrated to promote apoptosis and, therefore, DLC2 may serve as a ceRNA for Bcl-2 modifying factor (BMF) to promote apoptosis (31). The present study indicated that silencing of DLC2 in breast cancer resulted in changes of Rac-1, Cdc42 and Rock-2, therefore suggesting that not only RhoA, but also other RhoGTPases, may be involved in breast cancer. The specific underlying mechanisms and pathways involved require further investigation.

In conclusion, low expression of DLC2 in breast cancer tissue is associated with tumor differentiation, lymph node metastasis and poor prognosis. Loss of DLC2 promotes proliferation, migration and invasion of breast cancer cells, depending on the RhoGTPase pathway. These results indicate that DLC2 is a tumor suppressor in breast cancer. Future in vivo studies to confirm the results of the present in vitro experiments are scheduled to be performed.

\section{Acknowledgements}

Not applicable.

\section{Funding}

The present study was supported by the National Natural Science Foundation of China (grant no. 81102021), the Natural Science Foundation of Guangdong Province (grant no. 2014A030313032) and the Specialized Research Fund for the Doctoral Program of Higher Education (grant no. 20110171120076).

\section{Availability of data and materials}

The datasets used and analyzed during the present study are available from the corresponding author on reasonable request.

\section{Authors' contributions}

LX and YL designed this study. ZY performed the experiments and was a major contributor towards the writing of the manuscript. HC analyzed and interpreted the data. MS performed the immunohistochemistry experiments. YZ was responsible for the collection and analysis of clinical data. All authors read and approved the final manuscript.

\section{Ethics approval and consent to participate}

The First Affiliated Hospital of Sun Yat-sen University (Guangzhou, China) Ethics Committee approved the current study. All methods were performed in accordance with the relevant guidelines and regulations. All patients provided written informed consent prior to participation in the present study.

\section{Patient consent for publication}

Not applicable.

\section{Competing interests}

The authors declare that they have no competing interests.

\section{References}

1. Gray JM, Rasanayagam S, Engel C and Rizzo J: State of the evidence 2017: An update on the connection between breast cancer and the environment. Environ Health 16: 94, 2017.

2. Law AMK, Lim E, Ormandy CJ and Gallego-Ortega D: The innate and adaptive infiltrating immune systems as targets for breast cancer immunotherapy. Endocr Relat Cancer 24: X1, 2017.

3. Li Y, Li S, Meng X, Gan RY, Zhang JJ and Li HB: Dietary natural products for prevention and treatment of breast cancer. Nutrients 9: pii: E728, 2017.

4. Boix-Perales H, Borregaard J, Jensen KB, Ersbøll J, Galluzzo S, Giuliani R, Ciceroni C, Melchiorri D, Salmonson T, Bergh J, et al: The European medicines agency review of pertuzumab for the treatment of adult patients with HER2-positive metastatic or locally recurrent unresectable breast cancer: Summary of the scientific assessment of the committee for medicinal products for human use. Oncologist 19: 766-773, 2014.

5. Folkman J: Angiogenesis: An organizing principle for drug discovery. Nat Rev Drug Discov 6: 273-286, 2007.

6. Carmeliet P: Angiogenesis in health and disease. Nat Med 9: 653-660, 2003.

7. Ching YP, Wong CM, Chan SF, Leung TH, Ng DC, Jin DY and Ng IO: Deleted in liver cancer (DLC) 2 encodes a RhoGAP protein with growth suppressor function and is underexpressed in hepatocellular carcinoma. J Biol Chem 278: 10824-10830, 2003.

8. Vitiello E, Ferreira JG, Maiato H, Balda MS and Matter K: The tumour suppressor DLC2 ensures mitotic fidelity by coordinating spindle positioning and cell-cell adhesion. Nat Commun 5: 5826, 2014.

9. Chan FK, Chung SS, Ng IO and Chung SK: The RhoA GTPase-activating protein DLC2 modulates RhoA activity and hyperalgesia to noxious thermal and inflammatory stimuli. Neurosignals 20: 112-126, 2012.

10. Ullmannova V and Popescu NC: Expression profile of the tumor suppressor genes DLC-1 and DLC-2 in solid tumors. Int J Oncol 29: 1127-1132, 2006

11. Lin Y, Chen NT, Shih YP, Liao YC, Xue L and Lo SH: DLC2 modulates angiogenic responses in vascular endothelial cells by regulating cell attachment and migration. Oncogene 29: 3010-3016, 2010

12. Yakirevich E, Magi-Galluzzi C, Grada Z, Lu S, Resnick MB and Mangray S: Cadherin 17 is a sensitive and specific marker for metanephric adenoma. Am J Surg Pathol 39: 479-486, 2015.

13. Lakhani SR, Ellis IO, Schnitt SJ, Tan PH and van de Vijver MJ: WHO classification of tumours of the breast. Fourth edition. Int Agency Res Cancer, pp9-27, 2016.

14. Hanna S, Khalil B, Nasrallah A, Saykali BA, Sobh R, Nasser S and El-Sibai M: StarD13 is a tumor suppressor in breast cancer that regulates cell motility and invasion. Int J Oncol 44: 1499-1511, 2014.

15. El-Sitt S, Khalil BD, Hanna S, El-Sabban M, Fakhreddine N and El-Sibai M: DLC2/StarD13 plays a role of a tumor suppressor in astrocytoma. Oncol Rep 28: 511-518, 2012.

16. Leung TH, Yam JW, Chan LK, Ching YP and Ng IO: Deleted in liver cancer 2 suppresses cell growth via the regulation of the Raf-1-ERK1/2-p70S6K signalling pathway. Liver Int 30: 1315-1323, 2010.

17. Leung TH, Ching YP, Yam JW, Wong CM, Yau TO, Jin DY and Ng IO: Deleted in liver cancer 2 (DLC2) suppresses cell transformation by means of inhibition of RhoA activity. Proc Natl Acad Sci USA 102: 15207-15212, 2005.

18. Yuan BZ, Zhou X, Durkin ME, Zimonjic DB, Gumundsdottir K, Eyfjord JE, Thorgeirsson SS and Popescu NC: DLC-1 gene inhibits human breast cancer cell growth and in vivo tumorigenicity. Oncogene 22: 445-450, 2003.

19. Yuan BZ, Jefferson AM, Baldwin KT, Thorgeirsson SS, Popescu NC and Reynolds SH: DLC-1 operates as a tumor suppressor gene in human non-small cell lung carcinomas. Oncogene 23: 1405-1411, 2004.

20. Zhou X, Thorgeirsson SS and Popescu NC: Restoration of DLC-1 gene expression induces apoptosis and inhibits both cell growth and tumorigenicity in human hepatocellular carcinoma cells. Oncogene 23: 1308-1313, 2004.

21. Lukasik D, Wilczek E, Wasiutynski A and Gornicka B: Deleted in liver cancer protein family in human malignancies (Review). Oncol Lett 2: 763-768, 2011.

22. Kawai K, Kiyota M, Seike J, Deki Y and Yagisawa H: START-GAP3/DLC3 is a GAP for RhoA and Cdc42 and is localized in focal adhesions regulating cell morphology. Biochem Biophys Res Commun 364: 783-789, 2007. 
23. Zheng L, Li X, Chou J, Xiang C, Guo Q, Zhang Z, Guo X, Gao L, Xing Y and Xi T: StarD13 3'-untranslated region functions as a ceRNA for TP53INP1 in prohibiting migration and invasion of breast cancer cells by regulating miR-125b activity. Eur J Cell Biol 97: 23-31, 2018

24. Shih YP, Liao YC, Lin Y and Lo SH: DLC1 negatively regulates angiogenesis in a paracrine fashion. Cancer Res 70: 8270-8275, 2010 .

25. Tcherkezian $\mathrm{J}$ and Lamarche-Vane N: Current knowledge of the large RhoGAP family of proteins. Biol Cell 99: 67-86, 2007.

26. Petzold KM, Naumann H and Spagnoli FM: Rho signalling restriction by the RhoGAP Stard13 integrates growth and morphogenesis in the pancreas. Development 140: 126-135, 2013

27. Khalil BD, Hanna S, Saykali BA, El-Sitt S, Nasrallah A, Marston D, El-Sabban M, Hahn KM, Symons M and El-Sibai M: The regulation of RhoA at focal adhesions by StarD13 is important for astrocytoma cell motility. Exp Cell Res 321: 109-122, 2014.

28. Tang F, Zhang R, He Y, Zou M, Guo L and Xi T: MicroRNA-125b induces metastasis by targeting STARD13 in MCF-7 and MDA-MB-231 breast cancer cells. PLoS One 7: e35435, 2012.

29. Li X, Zheng L, Zhang F, Hu J, Chou J, Liu Y, Xing Y and Xi T: STARD13-correlated ceRNA network inhibits EMT and metastasis of breast cancer. Oncotarget 7: 23197-23211, 2016.
30. Hu J, Li X, Guo X, Guo Q, Xiang C, Zhang Z, Xing Y, Xi T and Zheng L: The CCR2 3'UTR functions as a competing endogenous RNA to inhibit breast cancer metastasis. J Cell Sci 130: 3399-3413, 2017.

31. Guo X, Xiang C, Zhang Z, Zhang F, Xi T and Zheng L: Displacement of Bax by BMFMediates STARD133'UTR-induced breast cancer cells apoptosis in an miRNA-depedent manner. Mol Pharm 15: 63-71, 2018.

32. Wolff AC, Hammond ME, Hicks DG, Dowsett $M$, McShane LM, Allison KH, Allred DC, Bartlett JM, Bilous M, Fitzgibbons $\mathrm{P}$, et al: Recommendations for human epidermal growth factor receptor 2 testing in breast cancer: American Society of Clinical Oncology/College of American Pathologists clinical practice guideline update. J Clin Oncol 31: 3997-4013, 2013.

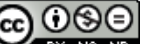

This work is licensed under a Creative Commons Attribution-NonCommercial-NoDerivatives 4.0 International (CC BY-NC-ND 4.0) License. 